\title{
EFEITO DO MICROCLIMA NA QUALIDADE DA UVA DE MESA 'ROMANA' (A 1105) CULTIVADA SOB COBERTURA PLÁSTICA ${ }^{1}$
}

\author{
JORGE LULU², JOSALBA VIDIGAL DE CASTRO³, MÁRIO JOSÉ PEDRO JÚNIOR ${ }^{4}$
}

\begin{abstract}
RESUMO - A videira é uma das principais fruteiras cultivadas em todo o mundo, e atualmente a preferência por uvas do tipo "sem sementes" ou "apirênicas" vem aumentando gradativamente no mercado interno brasileiro. A cultivar 'Romana' (A 1105) tem mostrado grande potencial como nova alternativa de uva de mesa apirênica na região de Jundiaí (SP). No entanto, a qualidade dos cachos tem sido afetada pela ocorrência de chuvas na época da colheita, propiciando a incidência de rachaduras nas bagas ("cracking") e podridões. Levando-se em consideração a viabilidade do cultivo desta uva sob cobertura plástica, foi realizada a presente pesquisa, objetivando-se analisar os parâmetros microclimáticos dentro e fora dos ambientes protegidos e a qualidade do produto na época de colheita. Os valores médios de transmissividade à radiação solar global entre os tratamentos foram decrescentes na seguinte ordem: efeito apenas da cobertura plástica $>$ efeito apenas do sistema de condução da planta $>$ efeito da cobertura plástica + sistema de condução da planta. Logo após a colheita, foram observadas reduções significativas na incidência de podridões, dano profundo e dano superficial nos cachos da videira cultivada sob cobertura plástica. A massa do cacho também foi significativamente maior no tratamento com cobertura plástica. Assim, pode-se concluir que o uso da cobertura plástica para o cultivo da uva 'Romana' (A 1105) foi capaz de melhorar a qualidade dos frutos, principalmente por reduzir significativamente a incidência de podridões nos cachos, importante problema que afetava esta cultivar.
\end{abstract}

Termos para indexação: Vitis sp., cultivo protegido, transmissividade, duração do período de molhamento, podridões, pós-colheita.

\section{EFFECT OF MICROCLIMATE IN THE QUALITY OF 'ROMANA' (A 1105) TABLE GRAPE CULTIVATED UNDER PLASTIC COVER}

\begin{abstract}
The grapevine is an important crop grown worldwide and nowadays the preference by seedless grapes is increasing in the brazilian market. The cultivar 'Romana' (A 1105) is showing great potential as a new seedless table grape for the growing region of Jundiaí, São Paulo, Brazil. However, the bunch quality has been influenced by rain during the harvest period, leading to incidence of berry cracking and rot. Aiming to verify the possibility of growing this table grape under plastic cover conditions a field trial was done in a vineyard grown in Jundiaí, aiming to quantify microclimatic parameters inside and outside of the protected environment; the bunch quality at harvest and the period of cold storage of bunchs (post-harvest time). The average values of solar radiation transmissivity were decreasing as follow: effect of the plastic cover only> effect of the grapevine training system only $>$ and combined effect of the plastic cover and training system. Analising the bunch quality parameters at harvest, significantly lower values of bunch rot were found for the plastic cover treatment in relation to the open sky grapevines. Also, the bunch weight was higher in the plastic cover treatment. The defects like deep and superficial damage were lower for the bunch harvested in the plastic cover treatment. So, it was concluded that the use of plastic cover for the 'Romana' (A 1105) table grape led to a better bunch quality, mainly due to the reduction of rot bunch incidence.
\end{abstract}

Index terms: Vitis sp.; plastic cover; transmissivity; leaf wetness duration; bunch rot; post-harvest.

\section{INTRODUÇÃO}

Ocupando atualmente uma área de 7,5 milhões de hectares, a videira é uma das principais fruteiras cultivadas no mundo, com uma produção anual de 62 milhões de toneladas (FAO, 2004), das quais 8,5 milhões são de uva para mesa. Analisando o mercado brasileiro de frutas de mesa, é possível perceber uma exigência cada vez maior dos consumidores nacionais por uvas de melhor qualidade, não somente em relação ao aspecto visual, mas também ao sabor, aroma e consistência, além de uma preferência por uvas do tipo "sem sementes" ou "apirênicas".

O INSTITUTO AGRONÔMICO (IAC) possui em seu banco de germoplasma diferentes variedades de uvas de mesa, dentre as quais a 'Romana' (A 1105), que é uma planta vigorosa e produtiva, com cachos médios a grandes, bem compactos e cônicos, bagas esverdeadas, médias a grandes, de textura crocante e com agradável sabor neutro (Pommer, 2001). A cultivar 'Romana' (A 1105) tem mostrado grande potencial como nova alternativa de uva de mesa apirênica na região de Jundiaí (SP). No entanto, de acordo com Souza \& Martins (2002), a qualidade dos cachos tem sido afetada pela ocorrência de chuvas na época da colheita, propiciando rachaduras nas bagas (“cracking") e incidência de podridões. Para evitar a chuva nos cachos na época da colheita e conseqüente melhoria de qualidade, o cultivo protegido surge como alternativa, principalmente tratando-se de uva fina de mesa.

Levando-se em consideração a viabilidade do cultivo da uva de mesa 'Romana' (A 1105) sob cobertura plástica, foi realizado o presente estudo na região de Jundiaí (SP), objetivando-se analisar as alterações das variáveis físicas do ambiente provocadas pelo cultivo protegido e seus efeitos na qualidade dos cachos na época de colheita.

\section{MATERIAL E MÉTODOS}

O ensaio foi feito no Centro Avançado de Pesquisa Tecnológica do Agronegócio de Frutas do INSTITUTO AGRONÔMICO (IAC), localizado no município de Jundiaí-SP (Lat.: $23^{\circ} 12^{\prime}$ S, Long.: $46^{\circ} 53^{\prime} \mathrm{W}$ e Alt.: $715 \mathrm{~m}$ ), durante um ciclo da cultura da videira, no ano-agrícola de 2003-04. A parcela experimental foi constituída de vinhedos da uva de mesa 'Romana' (A 1105), conduzidos em sistema de condução denominado cortina dupla ("Geneva Double Curtain”). O porta-enxerto utilizado foi o IAC 766 'Campinas', considerado bastante forte e

\footnotetext{
${ }^{1}$ (Trabalho 061/2005). Recebido: 08/04/2005. Aceito para publicação: 06/10/2005. Parte da dissertação do primeiro autor apresentada ao Instituto Agronômico (IAC) para a obtenção do título de Mestre em Agricultura Tropical e Subtropical na área de concentração Gestão de Recursos Agroambientais. Projeto financiado pela CAPES.

${ }^{2}$ Eng ${ }^{\circ}$ Agrícola, Doutorando, Universidade de São Paulo (USP), Escola Superior de Agricultura "Luiz de Queiroz" (ESALQ), Departamento de Ciências Exatas, Piracicaba - SP, (0XX19) 3429-4145 - Ramal: 222/231, e-mail: j_lulu@yahoo.com.

${ }^{3}$ Enga Agrônoma, Pesquisadora Científica, Prof. a Doutora, Instituto Agronômico (IAC), APTA, Campinas - SP, (0XX19) 3241-5188 - Ramal: 339, e-mail: josalba@iac.sp.gov.br.

${ }^{4}$ Eng $^{\circ}$ Agrônomo, Pesquisador Científico, Prof. Doutor, Instituto Agronômico (IAC), APTA, Campinas - SP, (0XX19) 3241-5188 - Ramal: 338, e-mail: mpedro@iac.sp.gov.br.
} 
vigoroso. As videiras foram plantadas num espaçamento de $3 \mathrm{~m}$ entre linhas (ruas) e de $1,5 \mathrm{~m}$ entre plantas, estando estas com idade de 8 anos. Já os tratos culturais e fitossanitários foram efetuados de acordo com as recomendações técnicas para a região.

Quando iniciou o período de chuvas mais intensas (04/12/ 2003) e os frutos da videira 'Romana' (A 1105) já entraram na fase de maturação (suscetível a ocorrência do "cracking” nas bagas), foi instalada uma cobertura plástica logo acima da copa das plantas, objetivando-se apenas evitar a ocorrência de chuva nos cachos. O formato do teto foi "em arco", com abertura nas laterais. O plástico utilizado foi um filme de polietileno de baixa densidade (PEBD), de 150 $\mu \mathrm{m}$ de espessura, transparente, com tratamento contra raios ultravioleta. Foram instaladas, ao acaso, coberturas plásticas em três ruas (parcelas) de $6 \mathrm{~m}$ de comprimento, com 4 plantas cada parcela, denominado tratamento "com cobertura plástica (CCP)". Em outras três parcelas, também escolhidas ao acaso, situavam-se as plantas do tratamento denominado "sem cobertura plástica (SCP)". O experimento foi conduzido no delineamento inteiramente casualizado, com 2 tratamentos, 3 repetições e 4 plantas por parcela.

No período de 04-12-2003 a 20-01-2004, foram feitos o monitoramento e a coleta dos dados microclimáticos interna e externamente ao vinhedo. A radiação solar global foi medida com radiômetros tubulares (marca Eltec) e a transmissividade à radiação solar global calculada em relação ao ambiente externo ao vinhedo. Já as temperaturas de bulbo seco e bulbo úmido do ar foram medidas com termopares de cobre-constantan, instalados em abrigos micrometeorológicos de ventilação natural. Por psicrometria, calculouse a umidade relativa do ar. Além disso, sempre que a umidade relativa do ar foi maior ou igual a $95 \%$, considerou-se a ocorrência de molhamento (orvalho), registrando assim a duração do período de molhamento (DPM), importante variável micrometeorológica para a estimativa de condições favoráveis à ocorrência de doenças fúngicas na videira, além do seu uso em sistemas de alerta fitossanitário, a fim de racionalizar o uso de fungicidas (Sentelhas, 2004). As medidas foram efetuadas na altura dos cachos de uva (no caso da radiação solar, também foram instalados sensores entre a copa da planta e a cobertura plástica). Os tratamentos CCP e SCP, de acordo com a localização dos sensores, foram subdivididos em: sensores instalados acima do dossel (entre o topo da planta e o plástico), denominados "com cobertura plástica acima (CCPc)"; sensores instalados abaixo do dossel (na altura dos cachos de uva), denominados "com cobertura plástica abaixo (CCPb)" e "sem cobertura plástica abaixo (SCPb)", e sensores instalados externamente ao vinhedo, denominados de "externo (EXT)". Os sensores foram conectados a um sistema de coleta automática de dados (marca Campbell Scientific, modelo CR 10), programado para realizar leitura a cada 1 minuto, obtendo-se médias diárias e valores extremos (máximos e mínimos).

A comparação das características de qualidade dos cachos entre os tratamentos CCP e SCP foi realizada no dia da colheita das uvas (21-01-2004). Foram colhidos 23 cachos de ambos os tratamentos. A avaliação dos cachos sem toalete foi efetuada quanto ao teor de sólidos solúveis totais, incidência de podridões e dano profundo. Estas características, quando fora dos limites de tolerância, são consideradas como defeitos graves, cuja incidência sobre a baga compromete sua aparência, conservação e qualidade, restringindo ou inviabilizando o consumo da uva (BRASIL, 2002). Foram também avaliados os defeitos leves, como dano superficial, degrana e queimado de sol, seguindo as normas do regulamento técnico de identidade e de qualidade para a classificação da uva fina de mesa (BRASIL, 2002), além da avaliação da massa dos cachos. A avaliação da massa dos cachos foi feita por pesagens individuais. O teor de sólidos solúveis totais foi determinado por meio da leitura direta em um refratômetro manual da marca Shibuya, de 0 a $32^{\circ}$ Brix, com escala de $0,2^{\circ}$ Brix. Essa determinação foi feita retirandose de cada cacho 4 (quatro) bagas, sendo uma da parte superior, duas da parte média e uma da parte inferior. Já a incidência de podridões, dano profundo, dano superficial, degrana e queimado de sol, foram analisadas por contagem do número de bagas com estes problemas, transformando-se os valores em porcentagem.

Os valores médios de radiação solar global $\left(\mathrm{Rg}_{\text {méd }}\right)$, transmissividade média à radiação solar global $\left(\mathrm{TR}_{\text {méd }}\right)$, temperatura máxima do ar $\left(\mathrm{T}_{\text {máx }}\right)$, temperatura mínima do $\operatorname{ar}\left(\mathrm{T}_{\text {min }}\right)$, temperatura média do $\operatorname{ar}\left(\mathrm{T}_{\text {méd }}\right)$, umidade relativa do ar $\left(\mathrm{UR}_{\text {méd }}\right)$ e duração do período de molhamento nos dias com e sem ocorrência de precipitação (DPM ${ }_{\text {méd dias }}$ e $D P M_{\text {méd dias sem chuva }}$ ), foram comparados pelo Teste-t (duas amostras presumindo variâncias equivalentes), de acordo com Vieira (1980). Os resultados das características de qualidade na colheita dos frutos dos tratamentos CCP e SCP foram comparados por meio de "análise de intervalos de confiança", como proposto por Snedecor \& Cochraw (1973).

\section{RESULTADOS E DISCUSSÃO}

Na Tabela 1, são apresentados os valores médios das variáveis microclimáticas avaliadas. Foram verificadas diferenças significativas de $\mathrm{Rg}_{\text {méd }}$ e $\mathrm{TR}_{\text {méd }}$ entre os tratamentos $\mathrm{CCPc}, \mathrm{CCPb}, \mathrm{SCPb}$ e ambiente externo (EXT). Observa-se que, no ambiente externo (EXT), ocorreram os maiores valores de $\mathrm{Rg}_{\text {méd, }}$, seguido respectivamente dos tratamentos $\mathrm{CCPc}$ (efeito apenas da cobertura plástica), $\mathrm{SCPb}$ (efeito apenas do sistema de condução da planta) e $\mathrm{CCPb}$ (efeito da cobertura plástica + sistema de condução da planta). Em relação à $T_{\text {máx }}, T_{\text {mín }}, T_{\text {méd }}, U_{R_{\text {méd }}}$, $\mathrm{DPM}_{\text {méd dias com chuva }}$ e DPM $\mathrm{méd}_{\text {dias sem chuva }}$, verificou-se que não houve diferenças significativas entre os tratamentos.

TABELA 1 - Valores médios da radiação solar global $\left(\operatorname{Rg}_{\text {méd }}\right)$, transmissividade $\left(\mathrm{TR}_{\text {méd }}\right)$, temperatura máxima $\left(\mathrm{T}_{\text {máx }}\right)$, temperatura mínima $\left(\mathrm{T}_{\text {min }}\right)$, temperatura média $\left(\mathrm{T}_{\text {méd }}\right)$, umidade relativa do ar ( (UR $\left._{\text {méd }}\right)$ e duração do período de molhamento nos dias com chuva (DPM méd dias com chuva $)$ e sem chuva (DPM méd dias sem chuva $)$, no ambiente externo (EXT), entre o topo da planta e o plástico $(\mathrm{CCPc})$, sem cobertura plástica, na altura dos cachos de uva $(\mathrm{SCPb})$ e com cobertura plástica, na altura dos cachos de uva (CCPb). Jundiaí-SP, 2003.

\begin{tabular}{|c|c|c|c|c|}
\hline & EXT & CCPc & SCPb & CCPb \\
\hline $\operatorname{Rg}_{\text {méd }}\left(\mathrm{MJ} \mathrm{m}^{-2}\right.$ dia $\left.^{-1}\right)$ & $23,8 \quad \mathrm{a}$ & $17,4 \mathrm{~b}$ & $11,5 \mathrm{c}$ & $8,3 \mathrm{~d}$ \\
\hline $\mathrm{TR}_{\text {méd }}(\%)$ & -------- & $73,1 \mathrm{a}$ & $48,3 \mathrm{~b}$ & $34,9 \mathrm{c}$ \\
\hline $\mathrm{T}_{\text {máx }}\left({ }^{\circ} \mathrm{C}\right)$ & 34,2 a & -------- & $34,4 \mathrm{a}$ & $34,3 \mathrm{a}$ \\
\hline $\mathrm{T}_{\text {mín }}\left({ }^{\circ} \mathrm{C}\right)$ & 20,9 a & ------- & $20,7 \mathrm{a}$ & $21,0 \mathrm{a}$ \\
\hline $\mathrm{T}_{\text {méd }}\left({ }^{\circ} \mathrm{C}\right)$ & 26,0 & -------- & $26,0 \mathrm{a}$ & $26,1 \mathrm{a}$ \\
\hline $\mathrm{UR}_{\text {méd }}(\%)$ & 86,8 & -------- & 86,8 a & $87,2 \mathrm{a}$ \\
\hline $\mathbf{D P M}_{\text {méd dias com chuva }}(\mathrm{h} / \mathrm{dia})$ & -------- & -------- & $13,6 \mathrm{a}$ & $12,1 \mathrm{a}$ \\
\hline $\mathbf{D P M}_{\text {méd dias sem chuva }}(\mathrm{h} /$ dia $)$ & -------- & -------- & 3,3 a & 4,5 a \\
\hline
\end{tabular}

Médias, dentro da mesma linha, seguidas pela mesma letra, não diferem entre si, pelo Teste-t (duas amostras presumindo variâncias equivalentes), a $5 \%$ de probabilidade.

Analisando a Tabela 2 e a Figura 1, verificou-se uma redução significativa na incidência de podridões no tratamento com cobertura plástica (média de $4,3 \%$ de podridões), em relação ao tratamento sem cobertura plástica (média de $34,5 \%$ de podridões). Este resultado mostra a eficiência do uso da cobertura plástica na redução do principal problema que vinha ocorrendo com a videira 'Romana' (A 1105) cultivada a céu aberto, ou seja, a incidência de podridões ocasionada pelo excesso de chuva no período da colheita dos frutos e maior DPM em dias com ocorrência de precipitação pluvial (Tabela 1). Com relação à ocorrência de dano profundo, que compromete o aspecto visual dos cachos, também foram observadas diferenças significativas entre os tratamentos com cobertura plástica (média de $2,1 \%$ de dano profundo) e sem cobertura plástica (média de 7,8\% de dano profundo). Também se verifica uma redução significativa na ocorrência de dano superficial no tratamento com cobertura plástica (média de 2,5\% de dano superficial) em relação ao tratamento sem cobertura plástica (média de 6,7\% de 


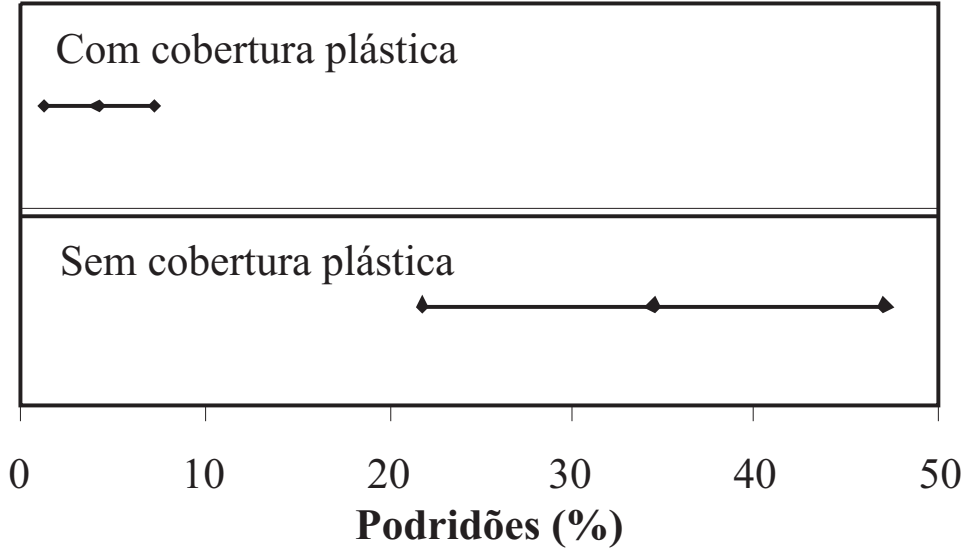

Podridões (\%)

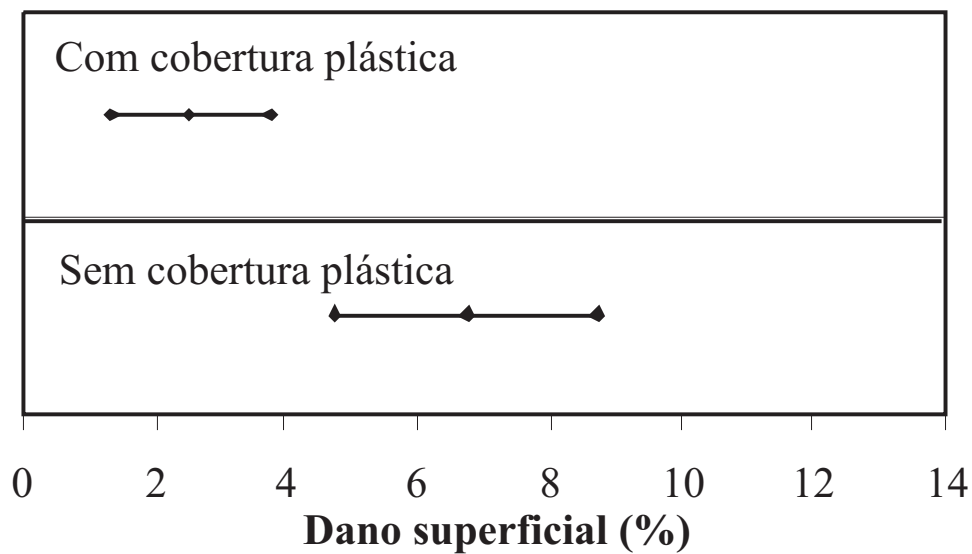

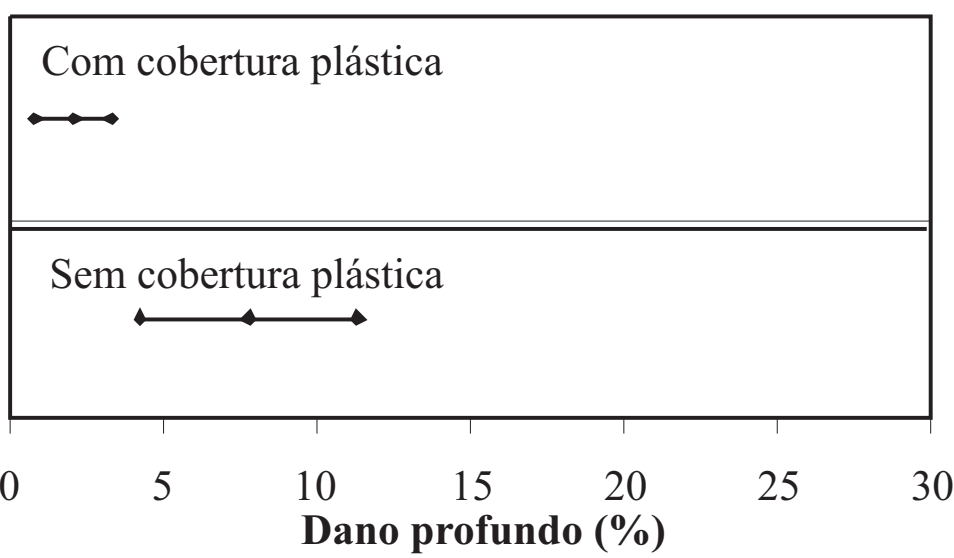

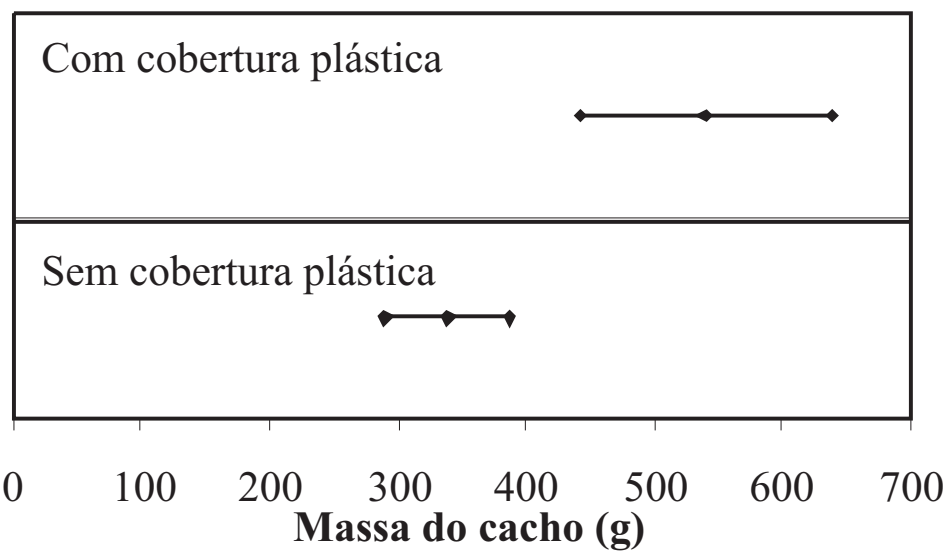

FIGURA 1 - Intervalos de confiança da incidência de podridões, dano profundo, dano superficial e da massa do cacho da uva de mesa 'Romana' (A 1105), sob os tratamentos com e sem cobertura plástica. Jundiaí-SP, 2004.

TABELA 2 - Valores médios e intervalos de confiança (IC) para as características de qualidade dos cachos (massa, teor de sólidos solúveis totais, podridões, dano profundo, dano superficial, degrana e queimado de sol), no dia da colheita das uvas (21-01-2004), nos tratamentos sem cobertura plástica (SCP) e com cobertura plástica (CCP). JundiaíSP, 2004.

\begin{tabular}{|c|c|c|c|}
\hline \multirow{2}{*}{$\begin{array}{c}\text { Características de qualidade do } \\
\text { cacho }\end{array}$} & & \multicolumn{2}{|c|}{ Tratamento } \\
\hline & & SCP & CCP \\
\hline Massa $(\mathrm{g})$ & $\begin{array}{c}\text { Média } \\
\text { IC }\end{array}$ & $\begin{array}{l}339,2 \mathrm{a} \\
\pm 48,4\end{array}$ & $\begin{array}{l}539,6 \mathrm{~b} \\
\pm 98,6\end{array}$ \\
\hline Teor de sólidos solúveis totais ( ${ }^{\circ}$ Brix) & $\begin{array}{l}\text { Média } \\
\text { IC }\end{array}$ & $\begin{array}{r}14,6 \quad \text { a } \\
\pm 0,5 \\
\end{array}$ & $\begin{array}{l}14,3 \text { a } \\
\pm 0,7\end{array}$ \\
\hline Podridões $(\%)$ & $\begin{array}{l}\text { Média } \\
\text { IC }\end{array}$ & $\begin{array}{l}34,5 \quad \text { a } \\
\pm 12,6\end{array}$ & $\begin{array}{l}4,3 \quad b \\
\pm 3,0\end{array}$ \\
\hline Dano profundo $(\%)$ & $\begin{array}{l}\text { Média } \\
\text { IC }\end{array}$ & $\begin{array}{ll}7,8 \quad \mathrm{a} \\
\pm 3,5 \\
\end{array}$ & $\begin{array}{l}2,1 \quad b \\
\pm 1,2 \\
\end{array}$ \\
\hline Dano superficial (\%) & $\begin{array}{l}\text { Média } \\
\text { IC }\end{array}$ & $\begin{array}{ll}6,7 \quad a \\
\pm 2,0\end{array}$ & $\begin{array}{l}2,5 \quad b \\
\pm 1,2\end{array}$ \\
\hline Degrana $(\%)$ & $\begin{array}{l}\text { Média } \\
\text { IC }\end{array}$ & $\begin{array}{ll}8,1 \quad a \\
\pm 4,5\end{array}$ & $\begin{array}{l}2,5 \quad \text { a } \\
\pm 1,8\end{array}$ \\
\hline Queimado de sol (\%) & $\begin{array}{l}\text { Média } \\
\text { IC }\end{array}$ & $\begin{array}{l}7,1 \quad a \\
\pm 6,3\end{array}$ & $\begin{array}{l}0,2 \quad \mathrm{a} \\
\pm 0,5\end{array}$ \\
\hline
\end{tabular}

Médias, dentro da mesma linha, seguidas pela mesma letra, não diferem entre si, pela "análise de intervalos de confiança", como proposto por Snedecor \& Cochraw (1973).

dano superficial). Estes resultados mostram a eficiência da cobertura plástica na proteção contra quaisquer tipos de danos mecânicos. Tais danos geralmente são decorrentes de adversidades climáticas, como chuva intensa, ventos fortes, granizo, etc., ou mesmo da entrada de pássaros ou insetos, sendo a cobertura plástica um meio de atenuar a ação destes agentes causadores do problema. A determinação da massa do cacho permitiu verificar que esta foi significativamente maior no tratamento com cobertura plástica (média de 539,6 g), em relação ao tratamento sem cobertura plástica (média de 339,2 g). Tal fato está diretamente relacionado com a incidência de podridões nos cachos do tratamento sem cobertura plástica, sendo que as bagas podres se tornam murchas e com massa bastante reduzida.

Pela Tabela 2, observou-se que não houve diferença significativa no teor de sólidos solúveis totais entre os frutos do tratamento com cobertura plástica (média de $14,3^{\circ}$ Brix) e os frutos do tratamento sem cobertura plástica (média de $14,6^{\circ} \mathrm{Brix}$ ). Estes valores situaram-se acima do teor mínimo exigido para uva fina de mesa, que é de $14^{\circ}$ Brix (BRASIL, 2002). Com relação à degrana, ou seja, bagas que se desprenderam dos engaços, considerada como defeito leve em uva fina de mesa, verificou-se que, no tratamento com cobertura plástica, a média foi de $2,5 \%$ de degrana e, sem cobertura plástica, média de $8,1 \%$ de degrana. Souza Leão (2002), para as variedades sem sementes 'Arizul' e 'Canner', também verificou degrana baixa, assim como no tratamento com cobertura plástica do presente experimento. Foi possível, portanto, verificar melhor comportamento, considerando-se a degrana nos frutos colhidos, do tratamento com cobertura plástica em relação ao tratamento sem cobertura plástica. $\mathrm{O}$ tratamento com cobertura plástica também apresentou resultados positivos quanto à redução do aparecimento de queimado de sol nos cachos da videira 'Romana' (A 1105). Observouse redução nos valores médios de ocorrência de queimado de sol no tratamento com cobertura plástica (média de $0,2 \%$ de queimado de sol) em relação ao tratamento sem cobertura plástica (média de $7,1 \%$ de queimado de sol). Estes resultados estão diretamente relacionados com a redução da transmissividade à radiação solar da cobertura plástica, conforme evidenciado na Tabela 1.

\section{CONCLUSÕES}

O uso da cobertura plástica para o cultivo da videira 'Romana' (A 1105) melhorou a qualidade dos frutos, principalmente por reduzir significativamente a incidência de podridões nos cachos, importante 
problema que afeta esta cultivar, tornando viável seu cultivo.

\section{REFERENCIAS}

FAO-FOOD AND AGRICULTURE ORGANIZATION OFTHE UNITED NATIONS. FAOSTAT-Agriculture. Disponível em: <http:// faostat.fao.org>. Acesso em: 08 mar. 2004.

BRASIL- Ministério da Agricultura, Pecuária e Abastecimento. Instrução normativa $\mathrm{n}^{\circ} 1 \mathrm{de} 1^{\circ}$ de fevereiro de 2002. Regulamento técnico de identidade e de qualidade para a classificação da uva fina de mesa. Diário Oficial da República Federativa do Brasil, Brasília, 04 fev. 2002, Seção $1.7 p$.

POMMER, C. V. Cultivares de uva produzidos ou introduzidos pelo IAC. In: SIMPÓSIO BRASILEIRO SOBRE UVAS DE MESA, 2000, Ilha Solteira. Anais... Ilha Solteira: FEIS - UNESP, 2001. p.51-67.
SENTELHAS, P.C. Duração do período de molhamento foliar: aspectos operacionais da sua medida, variabilidade espacial em diferentes culturas e sua estimativa a partir do modelo de Penman-Monteith. 2004; 161f. Tese (Livre-Docência em Agrometeorologia) Universidade de São Paulo, Escola Superior de Agricultura "Luiz de Queiroz", Universidade de São Paulo, Piracicaba, 2004. 161f.

SNEDECOR, G.W.; COCHRAW, W.G. Statistical methods. Iowa: Iowa State University, 1973. 593p.

SOUZA LEÃO, P.C. Comportamento de cultivares de uva sem sementes no submédio São Francisco. Revista Brasileira de Fruticultura, Jaboticabal, v.24, n.3, p.734-7, 2002.

SOUZA, J.S.I.; MARTINS, F.P. Viticultura brasileira: principais variedades e suas características. Piracicaba: FEALQ, 2002. 368p.

VIEIRA, S. Introdução à bioestatística. 3. ed. Rio de Janeiro: Elsevier, 1980. 196p. 